\title{
Study of The Spectral Response of A Terahertz Antenna Detector When The Surrounding Dielectric Changes
}

Mohammad Amin Bani

University of Kashan Faculty of Physics

Majid Nazeri (D M_Nazeri@kashanu.ac.ir)

University of Kashan Faculty of Physics https://orcid.org/0000-0002-9598-2179

Ahmad Sajedi bidgoli

University of Kashan Faculty of Physics

\section{Research Article}

Keywords: Terahertz, Detector antenna, Surface wave, Surrounding dielectric, CST

Posted Date: June 25th, 2021

DOI: https://doi.org/10.21203/rs.3.rs-621124/v1

License: (c) (1) This work is licensed under a Creative Commons Attribution 4.0 International License.

Read Full License 


\title{
Study of the spectral response of a terahertz antenna detector when the surrounding dielectric changes
}

\author{
Mohammad Amin Bani, Majid Nazeri, Ahmad Sajedi Bidgoli
}

\begin{abstract}
In this paper, the surface wave theory is used to study the spectral response of a terahertz antenna detector when the surrounding dielectric material is changed. As simulation shows, surrounding dielectrics affect the velocity of the current pulse propagation on electrodes. The propagation spectrum of the bow-tie and the dipole antenna on the LT-GaAs substrate is simulated through the FDTD method using the CST software. The current pulse generated in the antenna gap is also calculated with the Drude model. It is found that the type and the thickness of surrounding dielectrics affect the spectral responses of detector antennas as far as the peaks of the corresponding spectra shift down to one terahertz.
\end{abstract}

Keywords: , Terahertz, Detector antenna, Surface wave, Surrounding dielectric, CST

\section{Introduction}

The frequency region of an electromagnetic spectrum between microwave and infrared frequencies is called the terahertz band, which typically refers to the frequency range between 0.1 to 10 terahertz. Due to the rotational levels of many molecules in the terahertz range, absorption spectroscopy in this region provides unique information about the structure and chemical composition of molecules. Terahertz waves are of wide applications, and their generators and detectors have been studied vastly from the perspectives of theories, modelling as well as modern structures [1-4]. There are different methods for generating and detecting terahertz radiation. A few of the methods to produce terahertz radiation are the use of photoconductive antennas, optical rectification in non-linear media, oscillation in semi-conductive structures, and the use of quantum cascade lasers [5]. In this regard, photoconductive antennas, first proposed by Aston and Lee [6], are the most commonly used devices to generate and detect terahertz waves. The use of $\mathrm{THz}$ antennas is of lower cost and higher sensitivity than the other methods. These antennas usually consist of metal electrodes on low-temperature GaAs layers grown on SI-GaAs substrates and used to generate and detect terahertz radiation [7]. Simple setup, better signal-to-noise ratio, continuous bandwidth and room temperature performance are the best features of terahertz photoconductive antennas. In contrast, low frequency peaks and the low efficiency of converting optics to terahertz are the weaknesses of these antennas; their frequency peak is usually below $1 \mathrm{THz}$, and the efficiency is less than $0.1 \%$. Many efforts have been made to improve the responses and efficiency of antennas [8], such as numerical studies [9] using large aperture antennas [10] as well as the use of terahertz photoconductive antennas based on nano-structures and graphene and microlenses in antenna gaps [11, 12]. Of them, microlenses are used in array antennas and fiber-coupled antennas to simplify their setup and inhance their efficiency. It has been observed that the presence of a dielectric layer on an antenna affects the propagation of plasmonic waves on the electrodes and, in turn, the frequency response of the generating antenna [13-14]. 
This study aims to evaluate the effect of a dielectric surrounding the electrodes of a detector antenna, e.g. a bow-tie or dipole antenna, on the detector frequency response.

\section{Theoretical basis}

When a femtosecond laser pulse illuminates the antenna gap, electron-hole pairs are generated. If a DC voltage biases the electrodes, the generated photoelectrons are accelerated and a current pulse is propagated across the gap between the electrodes. Changes in the electric current in terahertz biased antennas induce electromagnetic radiation in the terahertz range (emitter). In terahertz detector antennas where there is no external bias voltage, a simultaneously illuminated $\mathrm{THz}$ field accelerates the photoelectrons generated by the fs optical pulse in the gap and creates a current that is proportional to the magnitude of the coupled terahertz field in the antenna [15]. The current density in the detector antenna is obtained based on the convolution of the optical pulse time pattern and the semiconductor properties. It is expressed by the following equation:

$$
j(t, \Delta t)=P_{o p t}(t) \otimes\left\{\exp \left(\frac{-t}{\tau_{\text {rec }}}\right) q v(t ; \Delta t)\right\}
$$

where $\Delta \mathrm{t}$ is the time delay between the terahertz pulse and the optical pulse, $P_{\text {opt }}$ is the optical pulse power, $\tau$ rec is the recombination time of the carriers, and $v$ is the average velocity of the carriers in the gap semiconductor [5, 10]. The generated current pulse propagates through the electrodes as a surface plasmonic wave. The amplitude and the propagation velocity of this pulse depend on the shape and the material of the electrodes. An analysis of the surface plasmon can help to predict the specifications of the $\mathrm{THz}$ antenna, emitter and detector [13]. In other words, an analysis of the surface wave propagation over the boundary of two semi-infinite media can give an analytic answer and draw a road map for the numerical analyses of antennas.

Figure 1 shows the boundary of two semi-infinite media. To investigate the propagation of terahertz waves in electrodes, it is first assumed that the terahertz pulse, in form of an electromagnetic wave with frequency $\omega$ that propagates in the $-\mathrm{z}$ direction, reaches the boundary of the two media. Then, this wave propagates as a surface wave on the common surface in the $\mathrm{x}$ direction. Maxwell equations can be used to find the longitudinal and transverse components of the field. In this structure, surface waves are subdivided into TE and TM polarization types. Since surface plasmon polarizations exist only for the TM mode, investigations are performed for this mode. The wave equations for $\mathrm{z}>0$ are as follows [16]:

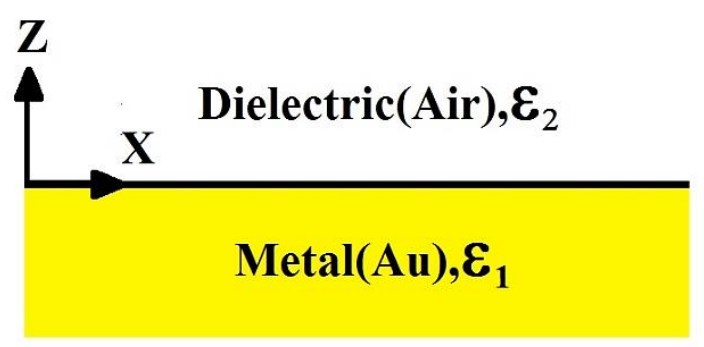

Figure 1. Geometry of a metal-dielectric junction 


$$
\begin{aligned}
& H_{y}(z)=A_{2} e^{i \beta x} e^{-k_{2} z} \\
& E_{x}(z)=i A_{2} \frac{1}{\omega \varepsilon_{0} \varepsilon_{2}} k_{2} e^{i \beta x} e^{-k_{2} z} \\
& E_{z}(z)=-A_{1} \frac{\beta}{\omega \varepsilon_{0} \varepsilon_{2}} e^{i \beta x} e^{-k_{2} z}
\end{aligned}
$$

and for $\mathrm{z}<0$ :

$$
\begin{aligned}
& H_{y}(z)=A_{1} e^{i \beta x} e^{-k_{1} z} \\
& E_{x}(z)=-i A_{1} \frac{1}{\omega \varepsilon_{0} \varepsilon_{1}} k_{1} e^{i \beta x} e^{-k_{1} z} \\
& E_{z}(z)=-A_{1} \frac{\beta}{\omega \varepsilon_{0} \varepsilon_{1}} e^{i \beta x} e^{-k_{1} z}
\end{aligned}
$$

where $k_{i}\left(k_{i} \equiv k_{z, i}, i=1,2\right)$ is the wave vector component perpendicular to the boundary of the two media and $\beta$ is the propagation constant. The value $\frac{1}{k_{z}}$ is defined as the penetration depth. The continuity of the electric field and the magnetic field can be included as a condition through the following equations:

$$
\begin{aligned}
& \frac{k_{2}}{k_{1}}=-\frac{\varepsilon_{2}}{\varepsilon_{1}} \\
& k_{1}^{2}=\beta^{2}-k_{0}^{2} \varepsilon_{1} \\
& k_{2}^{2}=\beta^{2}-k_{0}^{2} \varepsilon_{2}
\end{aligned}
$$

Through calculations, it is shown that the penetration depths of terahertz waves in a metal medium is much lower than the thickness of antenna electrodes, i.e. $100 \mathrm{~nm}$. Then, it can be assumed that the above solution is valid for plasmonic propagation on electrode layers. It is also concluded that the following propagation constant for surface plasmon has a good approximation and is valid for thin metal layers:

$$
\beta=k_{0} \sqrt{\frac{\varepsilon_{1} \varepsilon_{2}}{\varepsilon_{2}+\varepsilon_{1}}}
$$

The generated range of frequency is from 0.1 to 5 terahertz for the terahertz radiation. Therefore, when $\varepsilon_{1}=-877$ for $\mathrm{Au}$ and $\varepsilon_{2}=1$ for air, $\beta$ and $k_{1}$ are found to be of the values as reported in Table 1 .

As observed, because a pulse with a frequency range of 0.1 to 5 terahertz is used here, $k_{1}$ has different values for different frequencies. Therefore, the depth of the electric field penetration in the dielectric and the velocity of the wave propagation on the electrodes are different. This sometimes makes the dielectric layer change the overall shape of the terahertz pulse, and the effective length of the antenna will be different for different frequencies [16]. 
Table 1. Values of $\beta$ and $k_{1}$ for three different frequencies

\begin{tabular}{|c|c|c|}
\hline $\boldsymbol{f}$ & $\boldsymbol{\beta}$ & $\boldsymbol{k}_{\mathbf{1}}$ \\
\hline $0.1 \mathrm{THz}$ & 2094 & 70.7 \\
\hline $2.5 \mathrm{THz}$ & 52350 & 1767 \\
\hline $5 \mathrm{THz}$ & 104700 & 3534 \\
\hline
\end{tabular}

The next section discusses the effect of the dielectric layer on the antenna electrodes and clarifies how this layer in the form of a microlens may cause defects in the response of the antenna.

\section{Results and discussion}

The CST software was used to simulate the effects of the type and thickness of the surrounding dielectric on the detector antenna. First, a plane wave of a terahertz pulse was generated in the frequency range of 0.1 to $5 \mathrm{THz}$ with a peak of $2.74 \mathrm{THz}$. The simulated wave that illuminated the $\mathrm{THz}$ antennas consisted of $\mathrm{Au}$ electrodes layers on LT-GaAs in bow-tie and dipole shapes (Figure 2).

To study the effect of the surrounding dielectric on the detector antenna, the current generated in the antenna was simulated in two modes, with and without the dielectric layer at both bow-tie and dipole antennas. In this simulation, the surrounding dielectric layers were GaAs as a medium with a high dielectric coefficient (13) and silica as a typical dielectric with a dielectric constant of 3.84, which usually covers antennas as a microlens.
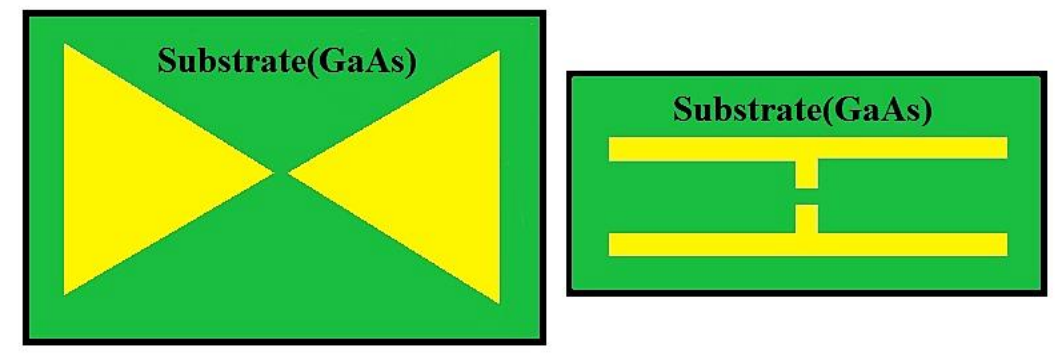

Figure 2. Designed terahertz detector antennas: (Left) bow-tie antenna and (Right) dipole antenna

The bow-tie antenna electrodes, which were triangular with the parameters of height $(\mathrm{H})$ and vertex angle $(\alpha)$, were designed in three sizes. They are presented in Figure 3 and Table 2 based on their $\mathrm{H}$ and $\alpha$ values. 
Table 2. The height $(\mathrm{H})$ and the angle of the vertex $(\alpha)$ are designed in three bow-tie antennas

\begin{tabular}{|c|c|c|}
\hline Type of antenna & $\boldsymbol{\alpha}$ & $\boldsymbol{H}$ \\
\hline Small & $26^{\circ}$ & $25 \mu \mathrm{m}$ \\
\hline Medium & $26^{\circ}$ & $75 \mu \mathrm{m}$ \\
\hline Large & $34^{\circ}$ & $100 \mu \mathrm{m}$ \\
\hline
\end{tabular}

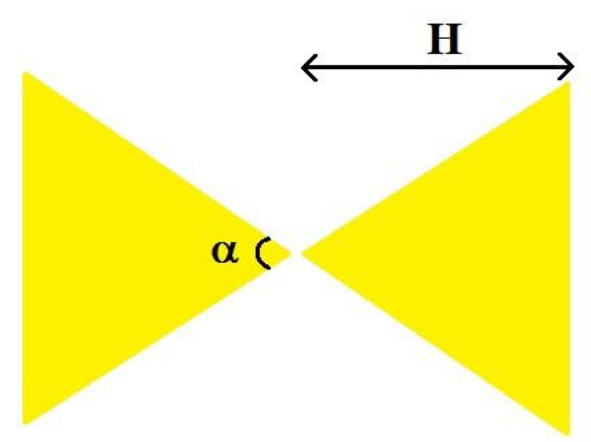

Figure 3. Vertex angle and height values in the terahertz bow-tie antenna electrodes 
At first, a signal was simulated for a large bow-tie antenna with no surrounding dielectric when it was illuminated by terahertz. Figure 4 shows the temporal and spectral curves of the emitted and detected signals.

The difference was due to the frequency behavior of the detector antenna, which caused the frequency peak of the detected signal to shift to lower frequencies. After this, a layer of GaAs dielectric was placed on the small detector antenna as a surrounding layer with a high dielectric coefficient, and the detected THz signals were studied when the antenna was illuminated with the same THz pulse.

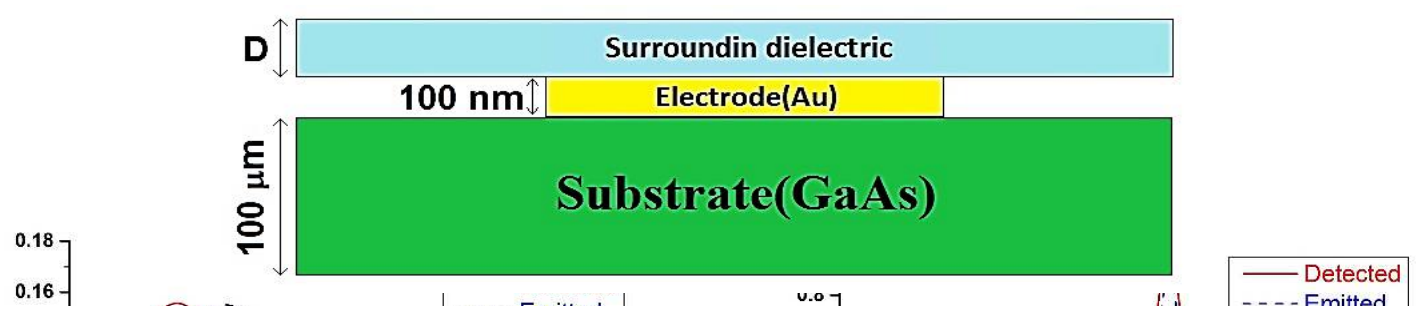

Figure 5. Geometry of the antenna electrodes between the GaAs substrate and the surrounding dielectric
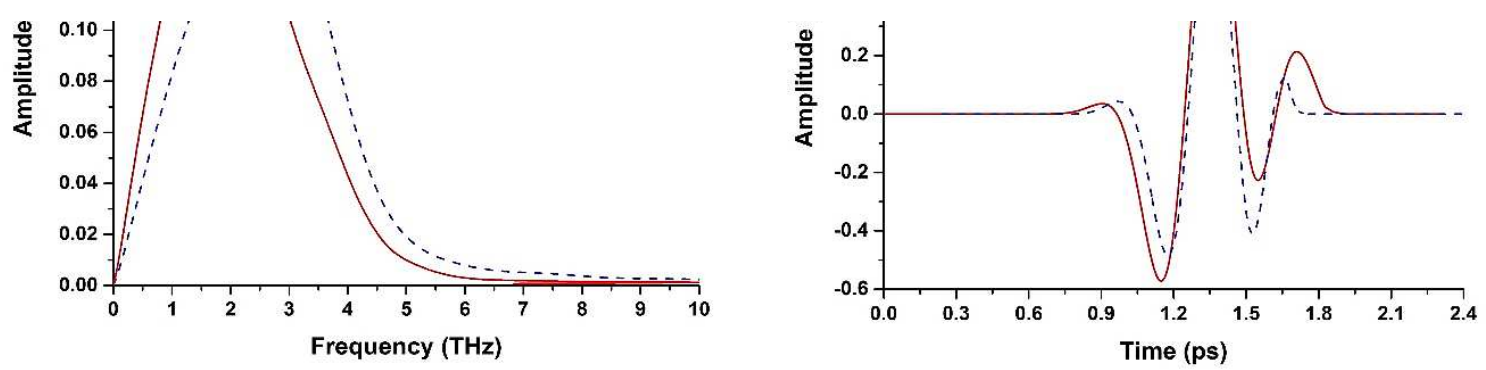

Figure 4. Diagram of the time response and the frequency response of the terahertz pulse for a large bow-tie antenna: (Right) the dash line is the time response of the emitted terahertz pulse, and the solid line is the time response of the detected terahertz pulse, (Left) the dash line is the frequency response of the emitted terahertz pulse, and the solid line is the frequency response of the detected terahertz pulse.

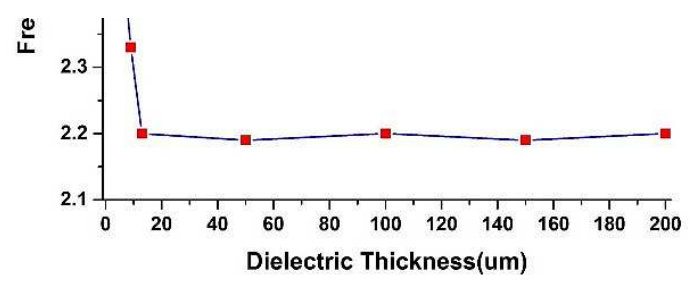

Figure 6. Changes of the frequency response peaks based on the changes in the surrounding dielectric thickness. Corner image: A small bow-tie antenna designed on a GaAs substrate with a thickness of $100 \mu \mathrm{m}$

The results of this simulation showed that, as the thickness of the GaAs surrounding layer increased, the antenna frequency response peak would shift to lower frequencies. In this antenna, the thickness of the 
surrounding dielectric layer (D) increased from 0 to $13 \mu \mathrm{m}$, which finally changed the frequency peak from 2.74 to 2.2 terahertz (Figure 6).

With further increase in the thickness of the surrounding dielectric, the resonance effects of Fabry Perot became observable in the frequency range of the radiation signal ( 0.1 to 5 terahertz). This phenomenon led to the formation of two or more frequency peaks in the detector response (Figure 7). However, as the thickness of the surrounding dielectric reached $200 \mu \mathrm{m}$, the frequency response peak of the antenna remained constant (Figure 6). 


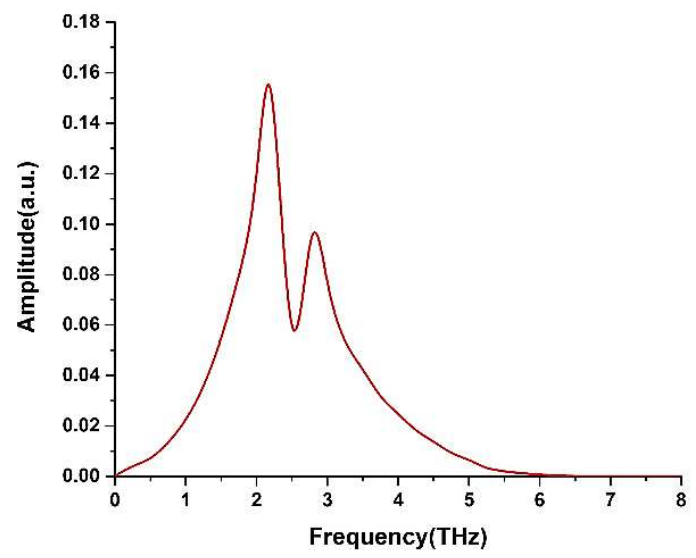

Figure 7. Appearance of a new peak with a frequency of 2.77 terahertz in the output signal of the bow-tie antenna due to the resonance effects of Fabry Perot

Most of the time, the dielectrics placed on antennas are in the form of silica microlenses placed on fiber coupled antennas or array antennas. Therefore, the next step of the simulation aimed at the effect of the surrounding silica dielectric on the small bow-tie antenna. As observed, an increase in the thickness of this type of surrounding dielectric on the antenna from 0 to $23 \mu \mathrm{m}$ led to the change of the frequency peak of the terahertz radiation from 2.74 to 2.35 terahertz.

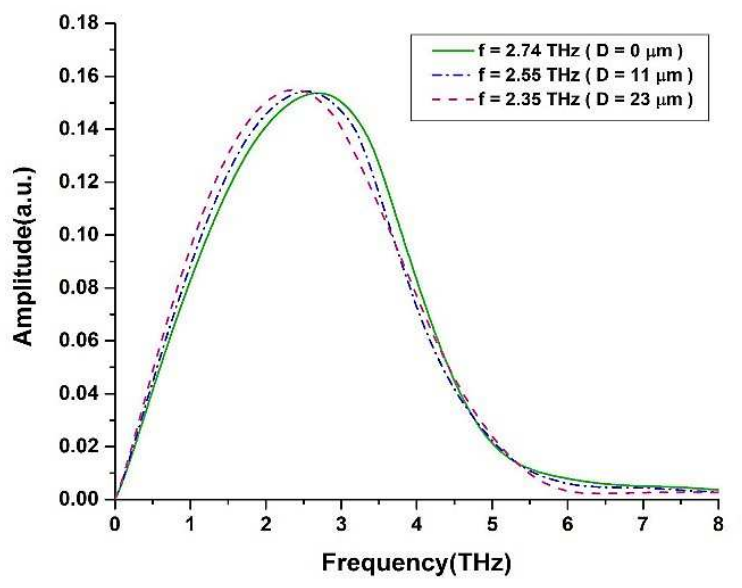

Figure 8. Frequency response of a small bow-tie detector antenna affected by the changes in the thickness of the surrounding silica dielectric on it: Three frequency response peaks of this antenna are shown in the figure. 
In the next part, medium and large antennas were used to check the accuracy of the simulation results. As the surrounding silica layer was placed on the medium antenna and the dielectric thickness was icreased to $38 \mu \mathrm{m}$, the output frequency of the antenna changed from 2.74 to 2.05 terahertz. It was also observed that placing a layer of silica on the large antenna and increasing the dielectric thickness to $44 \mu \mathrm{m}$ would make the peak frequency decrease from 2.74 to 1.68 terahertz.
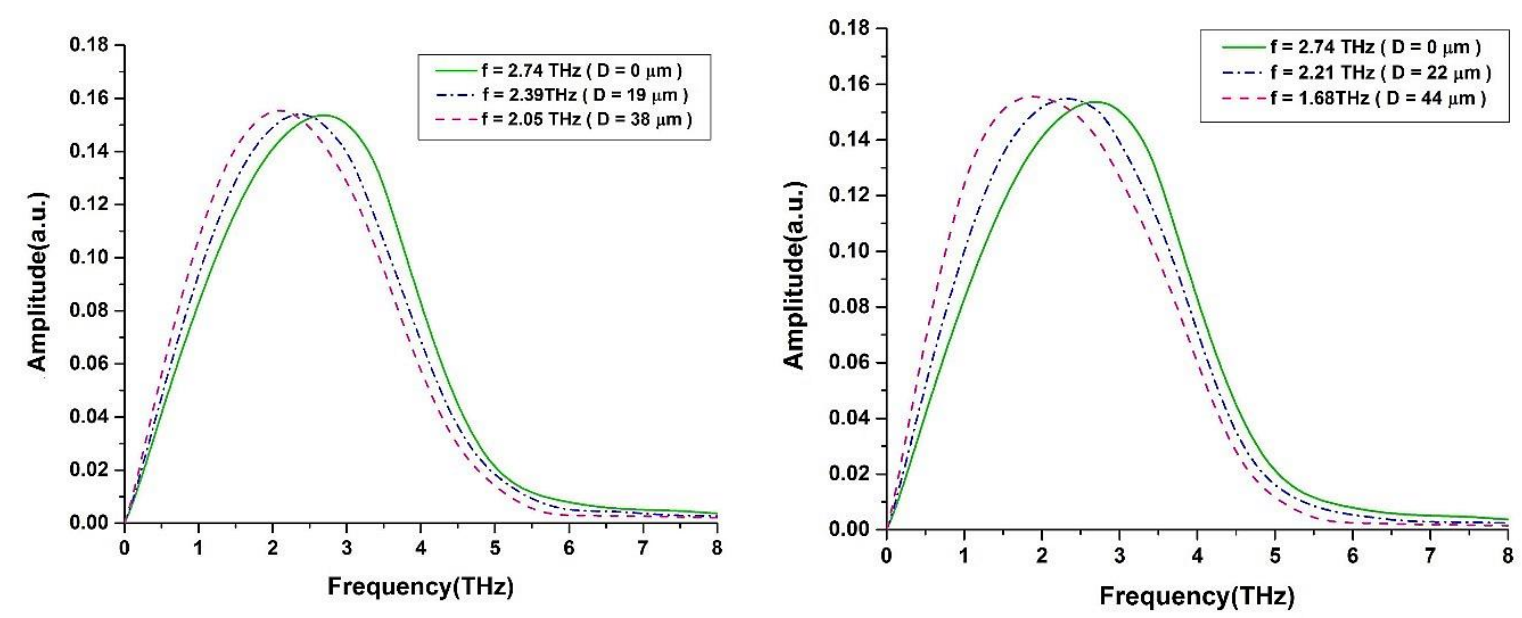

Figure 9. Frequency response of the bow-tie detector antenna affected by the changes in the thickness of the surrounding silica dielectric: (Left) three frequency response peaks for the medium antenna, and (Right) three frequency response peaks for the large antenna

As these simulations show, when the size of a bow-tie antenna is increased, the surrounding dielectric layer clearly affects the antenna response.

In the next part of the study, the shape of the antenna was changed and a dipole antenna was used instead of a bow-tie one. The dimensions of the dipole antenna were determined and simulated based on the parameters $\mathrm{m}, \mathrm{n}$ and $\mathrm{j}$, which related to different parts of the antenna electrodes in small, medium and large sizes, according to Figure 10 and Table 3.

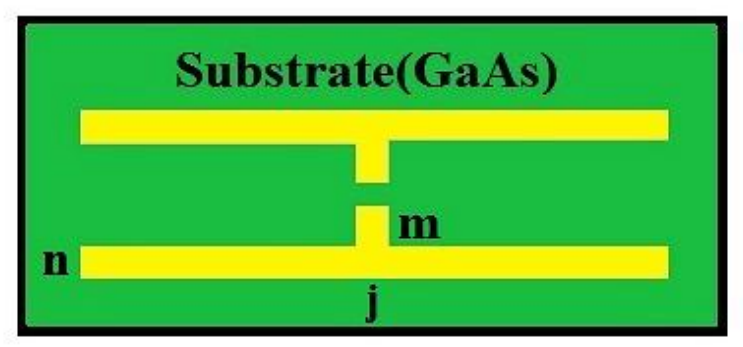

Figure 10. Dimensions of the dipole antenna used in the simulation 
Table 3. Parameters of the designed dipole antennas

\begin{tabular}{|c|c|c|c|}
\hline Type of antenna & $\boldsymbol{m}(\boldsymbol{\mu m})$ & $\boldsymbol{n}(\boldsymbol{\mu m})$ & $\boldsymbol{j}(\boldsymbol{\mu m})$ \\
\hline Small & 30 & 30 & 430 \\
\hline Medium & 50 & 50 & 430 \\
\hline Large & 80 & 80 & 430 \\
\hline
\end{tabular}

The frequency response of this type of antenna was investigated by the use of a silica dielectric. The response was affected by the changes in the dielectric layer. The simulation results showed that, when the terahertz pulse illuminated the small dipole antenna, the peak of the antenna frequency response decreased from 2.74 to 2.15 terahertz. This change was also induced by an increase in the dielectric thickness from 0 to $30 \mu \mathrm{m}$.

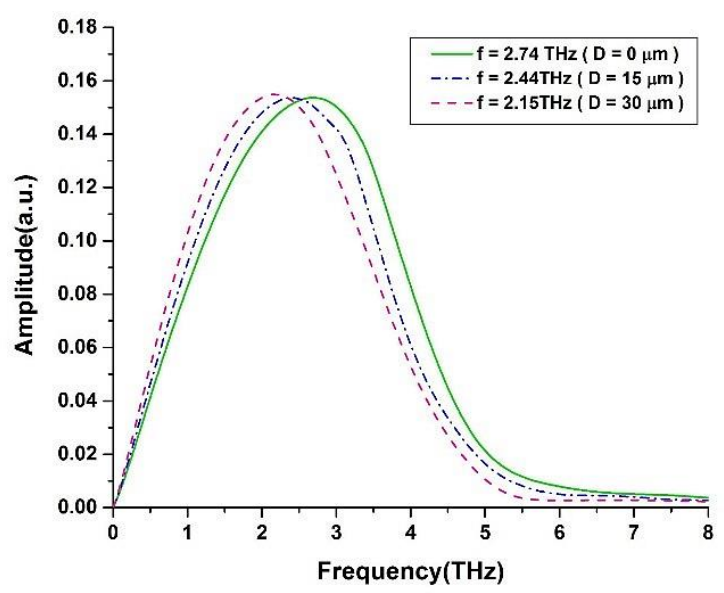

Figure 11. Change of the frequency response of the small dipole antenna due to the change in the thickness of the surrounding silica dielectric

Simulations were also performed for the other two sizes of dipole antennas (Figure 12). As shown in Figure 12 , once the thickness of the silica layer on the medium dipole antenna increased from 0 to $40 \mu \mathrm{m}$, the output frequency response of the antenna changed from 2.74 to 1.84 terahertz. In addition, for the large dipole antenna, the output frequency response changes from 2.74 to 1.55 terahertz as the thickness of the surrounding silica layer changed from zero to $47 \mu \mathrm{m}$. These simulation results are in agreement with those of the bow-tie antennas, emphasizing the role of the surrounding dielectric. 

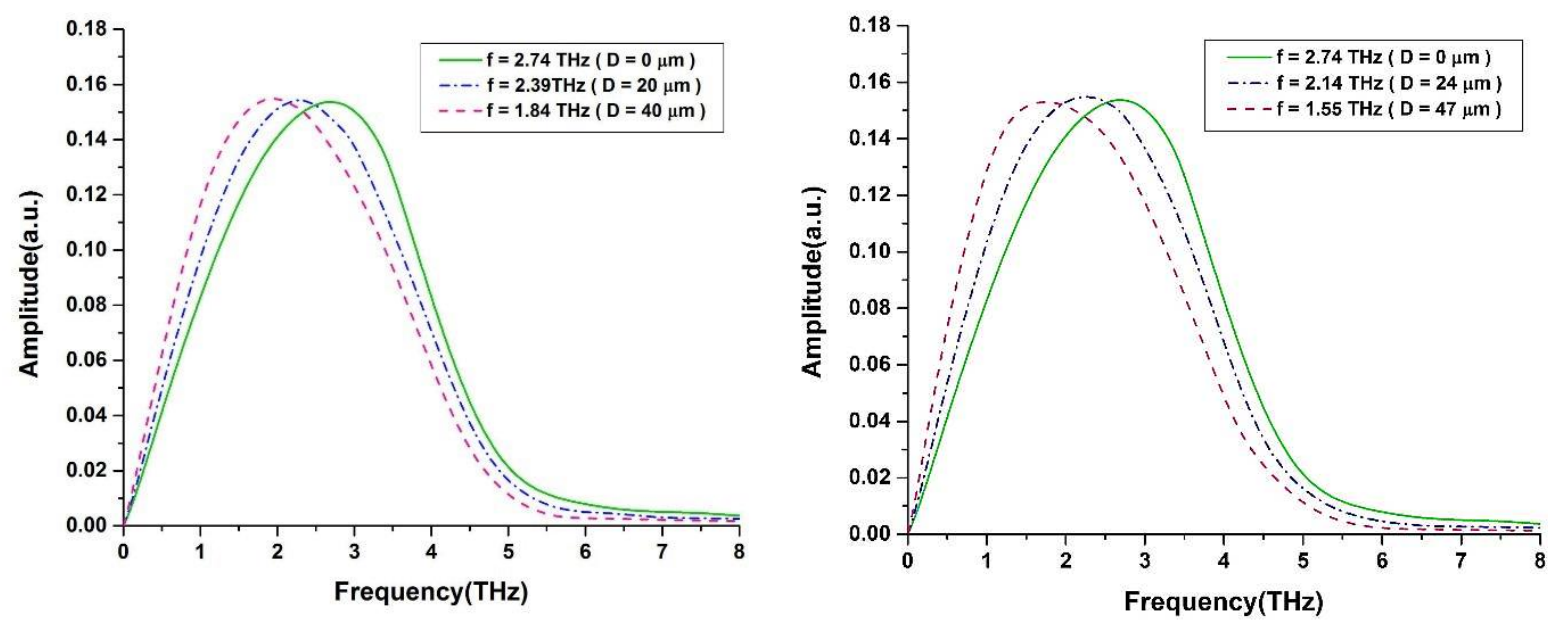

Figure 12. Frequency response of the dipole detector antenna based on the changes in the thickness of the surrounding silica dielectric: (Left) three frequency response peaks for the medium antenna, and (Right) three frequency response peaks for the large antenna

\section{Conclusion}

This study sought to explore how the change of the surrounding dielectric thickness would affect the frequency response of terahertz detector antennas. It was shown that covering a terahertz detector antenna with a layer of dielectric affects the response peak of the antenna; the thicker the dielectric, the lower the response peak. This is true of both bow-tie and dipole antennas whose electrodes are made of Au and are on LT-GaAs substrates. An increase in the dielectric thickness can also induce Fabry Perot resonance effects, in which case the spectral response of the antenna is accompanied with the main signal of another peak.

\section{References}

[1] Peiponen, Kai-Erik, Axel Zeitler, and Makoto Kuwata-Gonokami, eds. Terahertz spectroscopy and imaging. Vol. 171. Springer, 2012.

[2] Dexheimer, Susan L., ed. Terahertz spectroscopy: principles and applications. CRC press, 2017.

[3] Peiponen, Kai-Erik, Axel Zeitler, and Makoto Kuwata-Gonokami, eds. Terahertz spectroscopy and imaging. Vol. 171. Springer, 2012.

[4] Son, Joo-Hiuk, ed. Terahertz biomedical science and technology. CRC Press, 2014.

[5] Sakai, Kiyomi, and Masahiko Tani. "Introduction to terahertz pulses." Terahertz optoelectronics (2005): 1-30.

[6] Singh, Mandeep, and Simranjit Singh. "Design and Performance Investigation of Miniaturized MultiWideband Patch Antenna for Multiple Terahertz Applications." Photonics and NanostructuresFundamentals and Applications (2021): 100900. 
[7] Piao, Zhi-Sheng, Masahiko Tani, and Kiyomi Sakai. "Carrier dynamics and THz radiation in biased semiconductor structures." Terahertz Spectroscopy and Applications. Vol. 3617. International Society for Optics and Photonics, 1999.

[8] Kim, Joong H., Arup Polley, and Stephen E. Ralph. "Efficient photoconductive terahertz source using line excitation." Optics letters 30.18 (2005): 2490-2492.

[9] Zhang, Jitao. "Characterization of the terahertz photoconductive antenna by three-dimensional finitedifference time-domain method." arXiv preprint arXiv:1406.3872 (2014).

[10] Nazeri, M., and R. Massudi. "Study of a large-area THz antenna by using a finite difference time domain method and lossy transmission line." Semiconductor science and technology 25.4 (2010): 045007.

[11] Park, Sang-Gil, et al. "Enhancement of terahertz pulse emission by optical nanoantenna." ACS nano 6.3 (2012): 2026-2031.

[12] Zolfagharloo Koohi, M., and M. Neshat. "Evaluation of graphene-based terahertz photoconductive antennas." Scientia Iranica 22.3 (2015): 1299-1305.

[13] Nazeri, Majid, and Ahmad Sajedi Bidgoli. "Change of terahertz antenna spectrum when surrounding dielectric alters." Optik 183 (2019): 650-655.

[14] Berry, Christopher W., Mohammad R. Hashemi, and Mona Jarrahi. "Generation of high power pulsed terahertz radiation using a plasmonic photoconductive emitter array with logarithmic spiral antennas." Applied Physics Letters 104.8 (2014): 081122.

[15] Duvillaret, Lionel, et al. "Analytical modeling and optimization of terahertz time-domain spectroscopy experiments, using photoswitches as antennas." IEEE Journal of Selected Topics in Quantum Electronics 7.4 (2001): 615-623.

[16] Ghamsari, Behnood G., and A. Hamed Majedi. "Terahertz transmission lines based on surface waves in plasmonic waveguides." Journal of Applied Physics 104.8 (2008): 083108. 